\title{
A series of fortunate events: How the PDF method went from niche technique to mainstream and beyond
}

\author{
Simon J. L. Billinge \\ Department of Applied Physics and Applied Mathematics, Columbia University \\ And \\ Condensed Matter and Materials Science Department, Brookhaven National Laboratory
}

The origins of the atomic pair distribution function (PDF) technique go all the way back to the Debye Scattering equation in 1915 and its incorporation in the language of correlation functions through the work of Zernike and Prins in the 1920's. It was used somewhat as the method of last resort in the study of disordered systems and liquids, playing an important role but in a somewhat niche environment. With the advent of more powerful computers and new sources of radiation (time of flight neutrons in particular) and through the insights of previous Warren Award winner, Takeshi Egami, in the late 1980's and 1990's the method had a reawakening, being applied to study disorder in crystalline materials. Again, important impacts resulted, but it was still very much a niche and highly specialized technique, beloved of physicists (and not all of them by any means) but not too much beyond. Now the PDF technique is being widely adopted across disciplines from cultural heritage to earth science to catalysis, battery materials and pharmaceuticals. In fact, anywhere where disorder and nanoscale structures are valued in materials, the PDF is having an impact. And disorder and nanoscale structures are valued pretty much everywhere! There are multiple beamlines dedicated to the technique around the world, and software programs are becoming widely available and easier to use, easing access to the method. Moving forward we project the method's adoption and applications to grow enormously. In this talk I will describe, as I see it, the events that have given rise to this phenomenal growth. I will review where I think we are with the technique and the exciting possibilities that are just now emerging that will, hopefully be a launch-pad for even more impacts and growth in the power of the method and the scientific questions it can address. 